Psychonomic Monograph Supplements, 1967b, 2(7, Whole No. 23), 91-108.

SCHILLER, P. N., \& CHOROVER, S. L. Metacontrast: Its relation to evoked potentials. Science, 1966, 153, 1398-1401.

SIEGEL, S. Nonparametric statistics for the behavioral sciences. New York: McGraw-Hill, 1956.

WEISSTEIN, N. A Rashevsky-Landahl neural net: Simulation of metacontrast. Psychological Review, 1968, 75, 494-521. NOTE

1. This research was supported by the Physiological Psychology Program, Office of Naval Research, under ONR Contract No. N00014-67-A-0467-0009 and ONR Contract Authority No. NR $140-252$ to the first two authors.

\title{
Children's discrimination learning as a function of intertrial interval duration
}

\author{
WILLIAM L. CROLL, Institute of Child \\ Behavior and Development, University of \\ Iowa, Iowa City, Iowa 52240
}

Preschool children were trained on a successive discrimination in which four stimulus-response associations were learned concurrently, with different groups of $\mathrm{Ss}$ receiving ITI durations of $1,6,11$, and $16 \mathrm{sec}$. Learning rate was slower with a 1-sec duration than with a 6-, 11-, or 16-sec duration; but there was no difference in learning rate among the latter three durations. The results are compatible with the hypothesis that in such a task children do not benefit from opportunities to rehearse beyond several seconds because they do not retain the stimulus information needed for rehearsal.

The variables affecting children's discrimination learning have been extensively studied, but one potentially important variable, intertrial interval (ITI) duration, has been ignored. Several studies of adult concept utilization have found learning rate to depend upon ITI duration, suggesting that a similar effect might be obtained in children's discrimination learning (Bourne \& Bunderson, 1963; Bourne, Guy, Dodd, \& Justesen, 1965). Further suggestion of an ITI effect in children's discrimination learning is found in the effect of ITI duration on the accuracy of children's predictive behavior (Bogartz, 1967).

The present experiment investigated the effects of ITI duration in children's discrimination learning. Preschool children were trained on a successive discrimination in which four stimulus-response associations were learned concurrently, with different Ss receiving ITI durations of $1,6,11$, and $16 \mathrm{sec}$.

\section{METHOD}

The Ss were 68 children from the Preschool Laboratories of the Institute of Child Behavior and Development at the University of Iowa. They ranged in age from 55 to 69 months, with a mean of 63.4 months.

A translucent glass screen, 5 in. high and 12 in. wide, was mounted 33 in. above the floor on a wall of the experimental room. Stimuli were projected onto this screen by a slide projector recessed behind it. These stimuli were geometric forms, including a star, a rectangle, a diamond, and a circle. A horizontal array of four response buttons was centered 4 in. below the screen, with 2 in. between adjacent buttons. All stimulus events and time intervals were automatically controlled.

Each $\mathrm{S}$ was brought individually to the experimental room, shown a display of toys, and told that he could win one by playing a game. He was then seated before the screen and told: that pictures would appear on the screen, that each picture "belongs to" a button, and that he should press the button that he thought "belongs to" the picture being presented.

Each trial began with presentation of one of the four stimuli. When $S$ depressed any of the four buttons, the stimulus was immediately terminated, and $E$ immediately said "Yes" (if the response was correct) or "No" (if the response was incorrect). Stimulus offset initiated the ITI, which continued until onset of the stimulus for the next trial and during which the stimulus screen was blank. The Ss were assigned randomly to one of four ITI groups: 1, 6, 11, and $16 \mathrm{sec}$. Ss in a given ITI group received an ITI of that duration on each trial. The order in which the Ss were trained was balanced so that the number of $S s$ trained in one group never exceeded the number in any other group by more than two.

Each $S$ received 96 trials, 24 with each of the four stimuli. The order of stimulus presentations was random except that each of the four stimuli occurred exactly four times in each block of 16 trials, and no stimulus ever followed itself. The assignment of stimuli to response buttons was randomized across Ss.

\section{RESULTS}

The relative frequency of a correct response for each ITI group is given as a function of trial blocks in Table 1. An analysis of variance was performed on these scores, with ITI and trial blocks as factors. An .01 level of significance was employed for all tests. The interaction of ITI and trial blocks was significant $[F(15,320)=3.49]$ as was the effect of trial blocks $[\mathrm{F}(5,320)=35.30]$. The ITI effect was not significant $[F(3,64)=2.58]$. Table 1 suggests that the obtained interaction of ITI and trial blocks might have resulted from Groups 6, 11, and 16 increasing at a faster rate than Group 1. This was supported by two further analyses. The first compared the performance of Groups 6,11, and 16, deleting the data of Group 1. In this analysis, the ITI by Trial Blocks interaction was not significant $[F(10,240)=0.76]$, indicating that learning rate was the same for these groups. The trial blocks effect was again significant $[F(5,240)=30.39]$, and the ITI effect was again nonsignificant $[F(2,48)=0.13]$. The second analysis compared the data of Group 1 with the combined data of Groups 6,11 , and 16 . The ITI by Trial Blocks interaction was significant $[F(5,330)=6.39]$, suggesting that the learning rate of Group 1 was lower than that of the remaining groups. The trial blocks and ITI effects were both significant $[F(5,330)=27.08$ and $F(1,66)=7.66$, respectively].

\section{DISCUSSION}

The observed inferiority of performance under the 1-sec ITI duration is consistent with the results from adult concept-utilization experiments, but the failure to find a difference among the 6-, $11 \cdot$, and 16-sec ITI durations is

Table 1

Relative Frequency of a Correct Response as a Function of ITI Duration and Trial Blocks

\begin{tabular}{|c|c|c|c|c|}
\hline \multirow{2}{*}{$\begin{array}{l}\text { Trial } \\
\text { Block }\end{array}$} & \multicolumn{4}{|c|}{ ITI Duration (Sec) } \\
\hline & 1 & 6 & 11 & 16 \\
\hline 1 & .353 & .371 & .335 & .386 \\
\hline 2 & .379 & .515 & .463 & .533 \\
\hline 3 & .419 & .548 & .500 & .625 \\
\hline 4 & .401 & .629 & .563 & .662 \\
\hline 5 & .401 & .699 & .706 & .688 \\
\hline 6 & .353 & .765 & .706 & .662 \\
\hline
\end{tabular}


inconsistent with the results of these experiments. Bourne \& Bunderson (1963) trained different groups of adults with different ITI durations on a concept-utilization task and found that learning rate increased monotonically with ITI duration from 1 to $9 \mathrm{sec}$. Bourne et al (1965, Experiment 1) employed a wider range of ITI durations ( 1 to $25 \mathrm{sec}$ ) and found a nonmonotonic relation between learning rate and ITI duration. On a simple problem (one irrelevant dimension), learning rate increased with ITI from 1 to $9 \mathrm{sec}$, but additional increases in ITI produced a slight decrease in learning rate. On a complex problem. (five irrelevant dimensions), the maximum learning rate occurred at 17 rather than 9 sec.

Two processes have been identified that might produce the increase in learning rate with moderate increases in ITI found in the concept-utilization experiments (Bourne et al, 1965; Nodine, 1967). First, an increase in ITI duration increases the probability that $\mathbf{S}$ will have sufficient time to compare the currently presented concept instance with retained information from previously presented instances and then abstract from this information the stimulus properties that define the concept. The amount of time required for this processing is believed to be an increasing function of task complexity. Second, an increase in ITI provides greater opportunity for $S$ to rehearse since the ITI is typically unfilled.

In the present experiment, there were no irrelevant dimensions; thus, the amount of time required for comparison and abstraction is insignificant. The inferiority of performance for Group 1 is, therefore, more likely due to limitations on rehearsal than to limitations on comparison and abstraction. The failure to find learning-rate differences among ITI durations of 6,11 , and $16 \mathrm{sec}$ differs from the results of the Bourne et al experiments, and this is very likely due to differences in the Ss employed. Although the factors producing the performance decrement that occurred with long ITI durations in the Bourne et al experiments are not completely understood, there was some suggestion in Experiment 2 that it might have resulted from forgetting of stimulus information: When the stimulus remained present throughout the ITI, there was no performance decrement with long ITI durations. It might be expected that, for children, this forgetting of stimulus information would be even more pronounced. Bogartz (1967), for example, has reported that preschool children's retention of such information decreases considerably between 7.7 and $15.7 \mathrm{sec}$. Thus, the failure to find learning-rate differences among ITI durations of 6,11 , and $16 \mathrm{sec}$ in the present experiment may have resulted from $S s$ failing to recall sufficient stimulus information to enable rehearsal to facilitate performance.

\section{REFERENCES}

BOGARTZ, R. S. Extension of a theory of predictive behavior in young children to the effects of intertrial interval duration. Psychonomic Science, 1967, 8, 521-522.

BOURNE, L. E., JR., \& BUNDERSON, C. V. Effects of delay of informative feedback and length of postfeedback interval on concept identification. Journal of Experimental Psychology, 1963, 65, 1-5.

BOURNE, L. E., JR., GUY, D. E., DODD, D., \& JUSTESEN, D. R. Concept identification: The effects of varying length and informational components of the intertrial interval. Journal of Experimental Psychology, 1965, 69, 624-629.

NODINE, C. F. The role of temporal variables in the acquisition of concepts. In B. Kleinmuntz (Ed.) Concepts and the structure of memory. New York: Wiley, 1967, Pp. 227-239.

\section{ERRATUM}

McCALL, ROBERT B., Fels Research Institute, Yellow Springs, Ohio 45387, and MELSON, WILLIAM $H$. University of North Carolina, Chapel Hill, N. C. 27514. Psychonomic Science, 1969, 17 (6), $317-3 \mathrm{k} 8$. Attention in infants as a function of magnitude of discrepancy and habituation rate.- Page 317 , the lower half of Figure 1 was transposed. It is reproduced correctly in the two columns to the right.

Fig. 1. The stimuli used in the study. From left to right and from top to bottom, the stimuli are referred to in the text as $A$, $B, C$, and D, respectively.

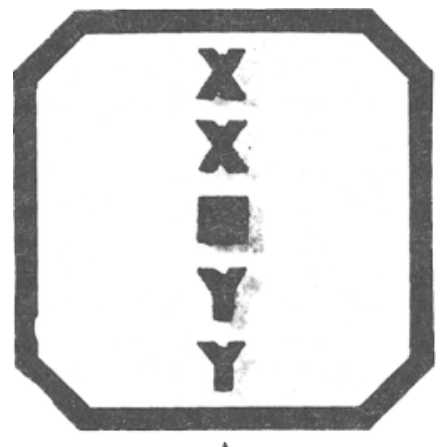

A

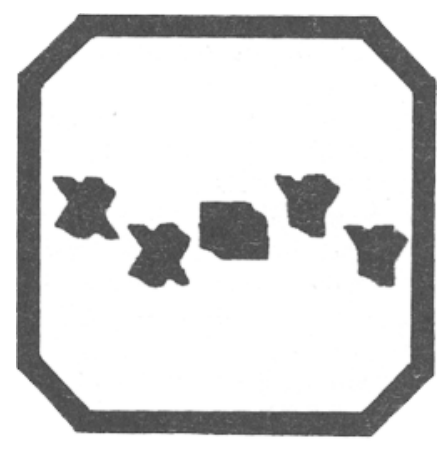

C

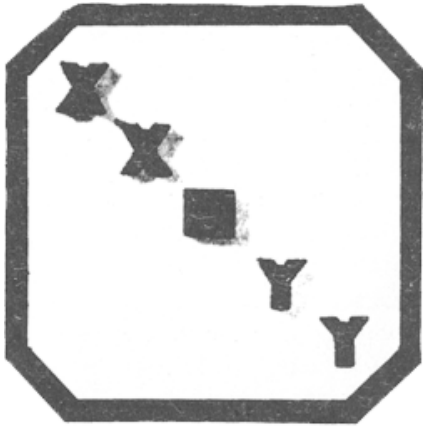

B

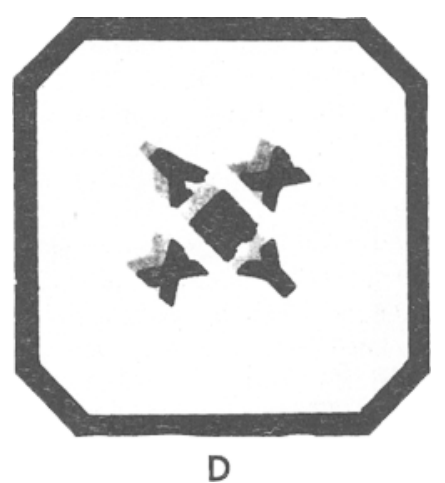

